\title{
Diagnostic yield of array comparative genomic hybridization in adults with autism spectrum disorders
}

\author{
Gary Stobbe, $\mathrm{MD}^{1,2}$, Yajuan Liu, $\mathrm{PhD}^{3}$, Rebecca $\mathrm{Wu}^{4}$, Laura Heath Hudgings, $\mathrm{MD}^{5}$, \\ Owen Thompson, $\mathrm{BS}^{6}$ and Fuki M. Hisama, MD ${ }^{1,7}$
}

Purpose: Array comparative genomic hybridization is available for the evaluation of autism spectrum disorders. The diagnostic yield of testing is $5-18 \%$ in children with developmental disabilities, including autism spectrum disorders and multiple congenital anomalies. The yield of array comparative genomic hybridization in the adult autism spectrum disorder population is unknown.

Methods: We performed a retrospective chart review for 40 consecutive patients referred for genetic evaluation of autism from July 2009 through April 2012. Four pediatric patients were excluded. Medical history and prior testing were reviewed. Clinical genetic evaluation and testing were offered to all patients.

Results: The study population comprised 36 patients (age range $18-45$, mean 25.3 years). An autism spectrum disorder diagnosis was confirmed in 34 of 36 patients by medical record review. One patient

\section{INTRODUCTION}

The autism spectrum disorders (ASDs) are neurodevelopmental conditions diagnosed in children with impairments in social relationships, language, and communication, along with restricted and repetitive behaviors. Many children with ASDs also have intellectual disability or epilepsy, and approximately $75 \%$ require lifelong social and behavioral support. One in 88 children has some form of ASD, and diagnosis rates have been rising in the past few decades. ${ }^{1,2}$ Therefore, autism is common and a reason for referral to geneticists, developmental pediatricians, and pediatric neurologists for diagnostic evaluation.

Technological advances have enabled the discovery of chromosomal copy-number changes and single-nucleotide changes on a genome-wide basis. ${ }^{3-5}$ Since these became available, many published studies have reported on the identification of copynumber changes in individuals with intellectual disability, developmental delay, or autism, and array comparative genomic hybridization (aCGH) has emerged as the single highest yield diagnostic test. The International Standard Cytogenomic Array Consortium reviewed 33 published studies of 21,698 patients with developmental delays, congenital anomalies, or autism had had an abnormal karyotype; none had prior array comparative genomic hybridization testing. Of the 23 patients with autism who underwent array comparative genomic hybridization, 2 of $23(8.7 \%)$ had pathogenic or presumed pathogenic abnormalities and 2 of 23 $(8.7 \%)$ had likely pathogenic copy-number variants. An additional 5 of $23(22 \%)$ of autism patients had variants of uncertain significance without subclassification.

Conclusion: Including one patient newly diagnosed with fragile $\mathrm{X}$ syndrome, our data showed abnormal or likely pathogenic findings in 5 of $24(21 \%)$ adult autism patients. Genetic reevaluation in adult autism patients is warranted.

Genet Med advance online publication 13 June 2013

Key Words: adult; array comparative genomic hybridization; autism; genetic testing; pervasive developmental disorders who were tested by aCGH and found an overall diagnostic yield of $12 \%$ in patients. ${ }^{6}$ Studies utilizing aCGH in predominantly pediatric cases of ASDs have yielded variable results, with the largest cohort (age range 19 months-22 years) demonstrating copy-number variants (CNVs) in 154 of 848 (18.2\%) of patients, including abnormal or possibly significant results in $7 \%$, with an additional $11.2 \%$ with variants of unknown significance. ${ }^{7}$

There are no studies of the diagnostic yield of aCGH in adults with ASD, although one study of 45 adult patients with intellectual disability reported a diagnostic yield of $22 \%$ and suggested the yield of aCGH may be higher in an adult as compared with a pediatric population. ${ }^{8}$ It could be hypothesized that the diagnostic yield of genetic testing in an adult ASD population would be lower than in a pediatric population because of childhood mortality from epilepsy or congenital anomalies. Alternatively, the yield could be higher in adults if chromosomal anomalies were not previously identified because of the limitations of prior testing or the lack of prior genetic evaluation. ${ }^{9} \mathrm{~A}$ third possibility is that the diagnostic yield is similar in adults and children but with a different distribution of abnormalities. The primary purpose of the present study was to assess the diagnostic yield of clinical genetic evaluation and aCGH in an adult ASD population.

\footnotetext{
The first two authors contributed equally to this work.

${ }^{1}$ Department of Neurology, University of Washington, Seattle, Washington, USA; ${ }^{2}$ Department of Psychiatry, University of Washington, Seattle, Washington, USA; ${ }^{3}$ Department of Pathology, University of Washington, Seattle, Washington, USA; ${ }^{4}$ Department of Neurobiology, University of Washington, Seattle, Washington, USA; ${ }^{5}$ Department of Family Medicine, University of Washington, Seattle, Washington, USA; ${ }^{6}$ Department of Genome Sciences, University of Washington, Seattle, Washington, USA; ${ }^{7}$ Division of Medical Genetics, Department of Medicine, University of Washington, Seattle, Washington, USA. Correspondence: Fuki M. Hisama (fmh2@u.washington.edu)
} 


\section{MATERIALS AND METHODS}

After approval by our institutional Human Subjects Review Board, a retrospective chart review was performed on all patients referred to the Autism Genetics Clinic in the Center for Human Development and Disability at the University of Washington from 1 July 2009 to 30 April 2012. A total of 40 patients were ascertained, including two male siblings. Four patients younger than 18 years were excluded. Medical record review confirmed the diagnosis of autism spectrum disorder in 34 of the 36 adult patients. Age; sex; developmental, education, and work history; prior genetic test results; neuroimaging; electroencephalograms; and medical history were reviewed. Prior genetic testing had been performed in 16 patients, and was nondiagnostic except in one patient $(6.25 \%)$ with a chromosome abnormality detected by karyotype. No patient had previously had aCGH testing.

Family history and physical exam were performed by a clinical geneticist and neurologist (F.M.H.). Fragile X molecular testing was offered if it had not been documented previously. aCGH was offered to all patients except patient 18 , in whom clinical suspicion of fragile $\mathrm{X}$ syndrome was high, so it was ordered as a first-line test. Because it was diagnostic, aCGH was not performed. Additional genetic testing was performed as clinically indicated and varied by patient.

The diagnosis of an ASD was based on Diagnostic and Statistical Manual of Mental Disorders, Fourth Edition, criteria and confirmed by chart review by a neurologist specializing in autism (G.S.). Twelve of the patients had undergone psychological testing. Although the testing battery varied depending on patient age, typical instruments included the Mini-Mental State Examination, Gilliam Asperger's Disorder Scale, Wechsler Adult Intelligence Scale-Third or Fourth Edition, Differential Ability Scales, Autism Diagnostic Observation Schedule, Behavioral Assessment System for Children, Beck Depression Inventory, and Beck Anxiety Scale. Results of cognitive testing were reviewed by a clinical psychologist blinded to the results of genetic testing.
Laboratory testing was performed by the Cytogenetics and Genomics Laboratory or the DNA Diagnostic Laboratory at the University of Washington Medical Center, unless otherwise stated.

Microarray comparative genomic hybridization analysis was performed using a whole-genome microarray with 135k oligonucleotide probes (NimbleGen CGX-3 v1.0; Roche NimbleGen, Madison, WI). Genomic DNA was extracted from peripheral blood using a Qiagen DNeasy Blood \& Tissue Kit (Qiagen, Valencia, CA). The concentration and quality of genomic DNA were determined using Spectrophotometer NanoDrop ND-1000 (Thermo Fisher Scientific, Wilmington, DE). Purified genomic DNA and normal control reference DNA were labeled with different fluorochromes using a Roche NimbleGen DualColor DNA Labeling Kit through random priming with Cy3 or Cy5 primers, and then co-hybridized onto the microarray slide. Hybridization and washing were performed as specified by the manufacturer. Microarray slides were scanned using an Agilent Microarray Scanner (Agilent Technologies, Santa Clara, CA). Whole-genome analysis of the CGH microarray was performed using NimbleScan 2.5 (Roche NimbleGen) and DNA Analytics 4.0 (Agilent Technologies, Santa Clara, CA). The global aberration detection method 1 algorithm with a threshold 10.0 and aberration filter for a minimum of five probes per region was applied. The $\log 2 \mathrm{R}$ ratios were calculated for information regarding copy number. The data and copy-number states were also visually inspected. Abnormal array results were confirmed by fluorescence in situ hybridization studies. Genomic linear positions for the copy-number changes (NCBI build 36, http:// genome.ucsc.edu/) are listed in Tables 1 and 2.

$\mathrm{CNVs}$ of uncertain significance (VUSs) were investigated by comparison with databases and by literature search. The databases that were used included the following: Database of Genomic Variants (DGV, http://projects.tcag.ca/variation/), Database of Structural Variation (dbVAR, http://www.ncbi. nlm.nih.gov/dbvar/), International Standard Cytogenomic

Table 1 Pathogenic and presumed pathogenic genetic syndromes diagnosed

\begin{tabular}{|c|c|c|c|c|c|c|c|c|}
\hline $\begin{array}{l}\text { Patient } \\
\text { number }\end{array}$ & $\begin{array}{l}\text { Chromosome } \\
\text { location }\end{array}$ & $\begin{array}{l}\text { Gain/ } \\
\text { loss }\end{array}$ & $\begin{array}{l}\text { Size } \\
(\mathrm{Mb})\end{array}$ & $\begin{array}{l}\text { Chromosome } \\
\text { coordinates (hg18) }\end{array}$ & Karyotype & Fragile X & Syndrome & Diagnosis \\
\hline 18 & Xq28 & NA & NA & NA & ND & $\begin{array}{l}270 \text { CGG } \\
\text { repeats, } \\
\text { hypermethylated }\end{array}$ & $\begin{array}{l}\text { Fragile } X \\
\text { syndrome }\end{array}$ & Autism \\
\hline 19 & $22 q 11.21$ & Loss & 2.49 & chr22:17,299,469-19,790,650 & ND & ND & $\begin{array}{l}\text { Velo-cardio-facial } \\
\text { syndrome }\end{array}$ & MCA, ID \\
\hline 25 & $17 p 11.2$ & Loss & 3.39 & chr17:16,755,641-20,147,193 & ND & ND & $\begin{array}{l}\text { Smith-Magenis } \\
\text { syndrome }\end{array}$ & PDD/NOS \\
\hline 26 & $2 q 31.3 q 36.1$ & Gain & NA & NA & $\begin{array}{l}\operatorname{dup}(2) \\
(q 31.3 q 36.1)\end{array}$ & Normal & NA & Autism \\
\hline 30 & $15 q 11.2 q 13.1$ & Gain & 5 & chr15:21,208,376-26,193,909 & Normal & Normal & $\begin{array}{l}\text { 15q11.2-q13 } \\
\text { Duplication } \\
\text { syndrome }\end{array}$ & Asperger \\
\hline 36 & $16 p 11.2$ & Loss & 0.535 & chr16:29,564,890-30,100,123 & ND & ND & $\begin{array}{l}16 p 11.2 \text { Deletion } \\
\text { syndrome }\end{array}$ & Schizoid \\
\hline
\end{tabular}

ID, intellectual disability; MCA, multiple congenital anomalies; NA, not available; ND, not done; PDD/NOS, pervasive developmental disorder, not otherwise specified. 
Table 2 Copy-number variants identified in adult autism patients

\begin{tabular}{|c|c|c|c|c|c|c|c|c|c|c|}
\hline Subject & $\begin{array}{l}\text { Chromosome } \\
\text { location }\end{array}$ & $\begin{array}{l}\text { Gain/ } \\
\text { loss }\end{array}$ & $\begin{array}{l}\text { Size } \\
\text { (kb, Mb) }\end{array}$ & $\begin{array}{l}\text { Chromosome } \\
\text { coordinates (hg18) }\end{array}$ & Karyotype & Fragile $\mathrm{X}$ & $\begin{array}{l}\text { Genes in } \\
\text { the region }\end{array}$ & Diagnosis & Interpretation & $\begin{array}{c}\text { Normal } \\
\text { controls } \\
(n=8,329) \\
(\%)\end{array}$ \\
\hline 4 & $4 q 24$ & Gain & $360 \mathrm{~kb}$ & $\begin{array}{l}\text { chr4:06,375, } \\
674-106,735,350\end{array}$ & ND & Normal & $\begin{array}{l}\text { TET2, PPA2, } \\
\text { EEF1A1P9, } \\
\text { ARHGEF38 }\end{array}$ & Asperger & VUS & 0 \\
\hline & $10 q 23.1$ & Loss & $45 \mathrm{~kb}$ & $\begin{array}{l}\text { chr10:84,130, } \\
199-84,175,730\end{array}$ & ND & Normal & $\begin{array}{l}\text { NRG3, } \\
\text { intronic }\end{array}$ & Autism & VUS & 0.07 \\
\hline 14 & $5 q 22.2$ & Loss & $100 \mathrm{~kb}$ & $\begin{array}{l}\text { chr5:112,270, } \\
214-112,315,453\end{array}$ & Normal & Normal & REEP5 & Autism & VUS & 0 \\
\hline 22 & $\begin{array}{l}\text { Xp22.33 or } \\
\text { Yp11.32 (PAR1) }\end{array}$ & Gain & $342.48 \mathrm{~kb}$ & $\begin{array}{l}\text { chrX:1,685, } \\
561-2,028,041\end{array}$ & ND & Normal & ASMT & Autism & VUS & NA \\
\hline 24 & Xp22.33 (PAR1) & Gain & $577.10 \mathrm{~kb}$ & $\begin{array}{l}\text { chrX:827,639-1,404, } \\
740\end{array}$ & ND & ND & $\begin{array}{l}\text { CRLF2, } \\
\text { CSF2RA }\end{array}$ & Autism & $\begin{array}{l}\text { VUS, likely } \\
\text { benign }\end{array}$ & NA \\
\hline 28 & 7p22.2-p21.3 & Gain & $4.67 \mathrm{Mb}$ & $\begin{array}{l}\text { chr7:3,612, } \\
717-8,278,510\end{array}$ & Normal & Normal & $\begin{array}{l}\text { FOXK1 } \\
\text { and } 48 \\
\text { additional } \\
\text { genes }\end{array}$ & Autism & $\begin{array}{l}\text { VUS, likely } \\
\text { pathogenic }\end{array}$ & 0 \\
\hline 29 & $7 q 31.1$ & Loss & $122 \mathrm{~kb}$ & $\begin{array}{l}\text { chr7:110,858, } \\
\text { 721-110,981,469 }\end{array}$ & Normal & Normal & IMMP2L & Autism & $\begin{array}{l}\text { VUS, likely } \\
\text { pathogenic }\end{array}$ & 0.56 \\
\hline 40 & Xp11.21 & Gain & $150 \mathrm{~kb}$ & $\begin{array}{l}\text { chrX:56,316, } \\
\text { 996-56,466,262 }\end{array}$ & Normal & Normal & KLF8 & Autism & VUS & NA \\
\hline
\end{tabular}

NA, not applicable; ND, not done; PAR1, chromosome X pseudoautosomal region 1; PDD/NOS, pervasive developmental disorder, not otherwise specified; VUS, variant of uncertain significance.

Normal controls refers to controls used in ref. 15 .

Array Consortium (https://isca.genetics.emory.edu), DatabasE of Chromosomal Imbalance and Phenotype in Humans using Ensembl Resources (DECIPHER, https://decipher.sanger. ac.uk/application/), OMIM (http://www.ncbi.nlm.nih.gov/ Omim), UCSC Genome Bioinformatics Site (http://genome. ucsc.edu/), and the European Cytogeneticists Association Register of Unbalanced Chromosome Aberrations (http:// www.ecaruca.net).

We followed the American College of Medical Genetics and Genomics Standards and Guidelines for interpretation of postnatal constitutional CNVs. ${ }^{10}$ This states that pathogenic CNVs will include well-documented syndromes and most cytogenetically visible alterations $(>3-5 \mathrm{Mb})$ unless the CNV corresponds to a well-known heteromorphism. Categorization as a CNV of uncertain clinical significance, likely pathogenic, is recommended if there is a prior single case report with well-defined breakpoints and phenotype, or a gene in the CNV interval is compelling and specific for the phenotype. Categorization as a $\mathrm{CNV}$ of uncertain clinical significance, likely benign, is recommended if there are no genes in the interval, or it is reported in a small number of cases in databases of variation in the general population but is not a common polymorphism. Categorization as a $\mathrm{CNV}$ of uncertain clinical significance (no subclassification) is recommended if there are genes in the interval, but it is unknown whether they are dosage sensitive, or the CNV is reported in multiple contradictory publications or databases.

\section{RESULTS}

\section{Clinical and demographic features}

Demographic characteristics for the 36 adult patients (28 men, 8 women) are presented in Table 3 . The average age was 25.3 years (range 18-45 years). Sixteen patients had had prior genetic testing, with a diagnostic abnormality in only one patient $(6.25 \%)$. Patient 22 is a 21 -year-old man who had a karyotype when he was 10 years old, which showed a partial duplication of the long arm of chromosome 2, reported as 46,XY,rec(2)dup(2)(q31.3q36.1)ins(2)(q33.1q31.3q36.1). His family declined aCGH testing.

Of the 36 patients, 25 patients had aCGH testing, including patients 19 and 36, who were referred for genetic evaluation of autism but for whom record review did not confirm the diagnosis. One patient had fragile X but not aCGH testing. Of 10 patients 
Table 3 Patient demographics for referrals for adults with autism from 1 July 2009 to 30 April 2012

\begin{tabular}{|c|c|}
\hline & Study cohort \\
\hline Total patients & 36 \\
\hline Mean age (years) & 25.3 (range: $18-45$ years) \\
\hline Gender, male:female & $3.5: 1$ \\
\hline Male (\%) & $28(78)$ \\
\hline Female (\%) & $8(22)$ \\
\hline \multicolumn{2}{|l|}{ Ethnicity (\%) } \\
\hline White & $29(80.6)$ \\
\hline Hispanic & $1(2.8)$ \\
\hline Black & $2(5.6)$ \\
\hline $\begin{array}{l}\text { Other (1 South Asian, } 3 \text { multicultural: } \\
2 \text { white/Asian, } 1 \text { Hispanic/white) }\end{array}$ & $4(11)$ \\
\hline \multicolumn{2}{|l|}{ Education (\%) } \\
\hline High school & $30(83)$ \\
\hline Technical school & $2(5.6)$ \\
\hline 2-Year college & $2(5.6)$ \\
\hline 4-Year college & 0 \\
\hline Unknown & $2(5.6)$ \\
\hline \multicolumn{2}{|l|}{ Residence (\%) } \\
\hline Parents/family & $25(69)$ \\
\hline Group home & $9(25)$ \\
\hline Semi-independent & $1(2.8)$ \\
\hline Independent & $1(2.8)$ \\
\hline \multicolumn{2}{|l|}{ ASD diagnosis (\%) } \\
\hline Autistic disorder & $26(72)$ \\
\hline Asperger disorder & $3(8.3)$ \\
\hline $\mathrm{PDD} / \mathrm{NOS}$ & $5(14)$ \\
\hline None & $2(5.6)$ \\
\hline \multicolumn{2}{|l|}{ Secondary diagnoses (\%) } \\
\hline Intellectual disability/number tested & $10 / 12(83)$ \\
\hline Epilepsy & $11(31)$ \\
\hline Dysmorphic (three or more anomalies) & $9(25)$ \\
\hline
\end{tabular}

ASD, autism spectrum disorder; PDD/NOS, pervasive developmental disorder, not otherwise specified.

not tested, 6 had insurance that did not cover aCGH testing, 3 declined because of the necessity for sedation for phlebotomy, and 1 declined because of a previous abnormal karyotype.

Of a total of 25 adult autism patients, 1 was diagnosed with fragile X syndrome, 1 had an abnormal karyotype, and 23 underwent aCGH testing. Only 12 confirmed ASD individuals had adequate psychological testing to determine intellectual status, of which 10 had at least borderline intellectual impairment. In addition, 9 autism patients had three or more minor anomalies and were considered dysmorphic. Of the 7 dysmorphic autism patients who underwent updated testing, 3 of 7 (43\%) had pathogenic findings and 1 of 7 had two VUSs identified. Epilepsy was reported in 11 individuals with autism; of these, 9 individuals underwent aCGH testing, 5 of $9(56 \%)$ had normal results, and 4 of 9 (44\%) had a VUS, all involving the $\mathrm{X}$ chromosome (Table 2).

\section{Pathogenic genetic test results}

Two patients were referred with a diagnosis of autism that could not be confirmed; however, aCGH was performed in them for other indications. Patient 19 had no neuropsychological testing or medical records available to confirm autism. He is a 26-yearold man who was born with cleft palate, congenital heart defect, and imperforate anus. The diagnosis of velo-cardio-facial syndrome was considered clinically, and aCGH was performed for congenital anomalies and intellectual disability. He had a $2.40 \mathrm{Mb}$ deletion on chromosome 22q11.21, confirming velo-cardiofacial syndrome. Patient 36 is a 19 -year-old man with intellectual disability and dysmorphic features. He met Diagnostic and Statistical Manual of Mental Disorders, Fourth Edition, criteria for schizoid personality disorder and rate-disabled reading disorder and has a chromosome 16p11.2 deletion, which been reported in approximately $1 \%$ of individuals with autism. ${ }^{11,12}$ Of the 24 autism patients who had current genetic testing, fragile X DNA testing identified a fully methylated, trinucleotide repeat expansion (270 CGG repeats) in patient 18 . He is a 45 -year-old man with intellectual disability, autism, macrocephaly, large ears, long face, prominent chin, macro-orchidism, and a maternal uncle with intellectual disability.

Of the 23 patients with confirmed autism who underwent aCGH, results were abnormal and pathogenic or presumed pathogenic in 2 of 23 patients (8.7\%). One patient was found to have Smith-Magenis syndrome, and one patient was diagnosed with 15q11.2-q13 duplication. The 15q11.2-q13 duplication in the patient includes the entire Prader-Willi/Angelman syndrome critical region, including the SNRPN gene. This duplication has been associated with autism and development delay, usually when it is present on the maternally inherited chromosome 15, although there are rare reports of the duplication arising on the paternal chromosome 15. ${ }^{13,14}$ This patient's parents declined follow-up aCGH studies. Given her presentation, this duplication was probably on the maternally inherited or derived chromosome 15 and presumed to be pathogenic. The results and clinical features are summarized in Tables 1 and 4.

\section{Analysis of VUSs}

We identified rare CNVs in 9 of 23 (39\%) autism patients, and two (patients 6 and 14) had both a copy gain and a copy loss. The genetic test results are summarized in Table 2, and clinical features are summarized in Table 5. Each VUS was evaluated to see how well it was represented in the DGV, and its frequency was calculated using a control cohort (8,329 unaffected adults) in dbVar from a previously published study that used an array platform related to that used by the patients in this study, whereas the controls were studied on a higher-resolution platform..$^{15}$ These CNVs are rare, and their frequencies in this normal control cohort ranged from 0 to $0.56 \%$ (Table 2). 
Table 4 Summary of clinical features for the patients in Table 1

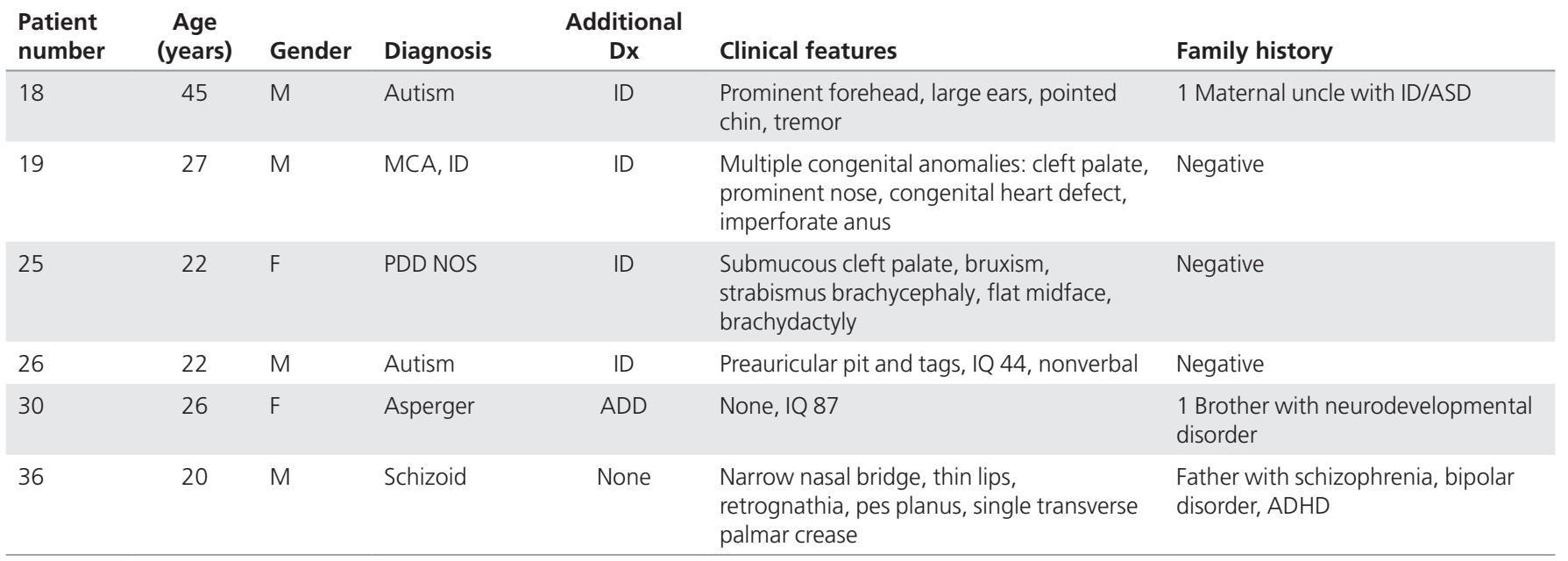

ADD, attention-deficit disorder; ADHD, attention-deficit hyperactivity disorder; ASD, autism spectrum disorder; Dx, diagnosis; F, female; ID, intellectual disability; IQ, intelligence quotient; $\mathrm{M}$, male; MCA, multiple congenital anomalies; PDD/NOS, pervasive developmental disorder, not otherwise specified.

Table 5 Summary of clinical features in adults with aCGH VUS

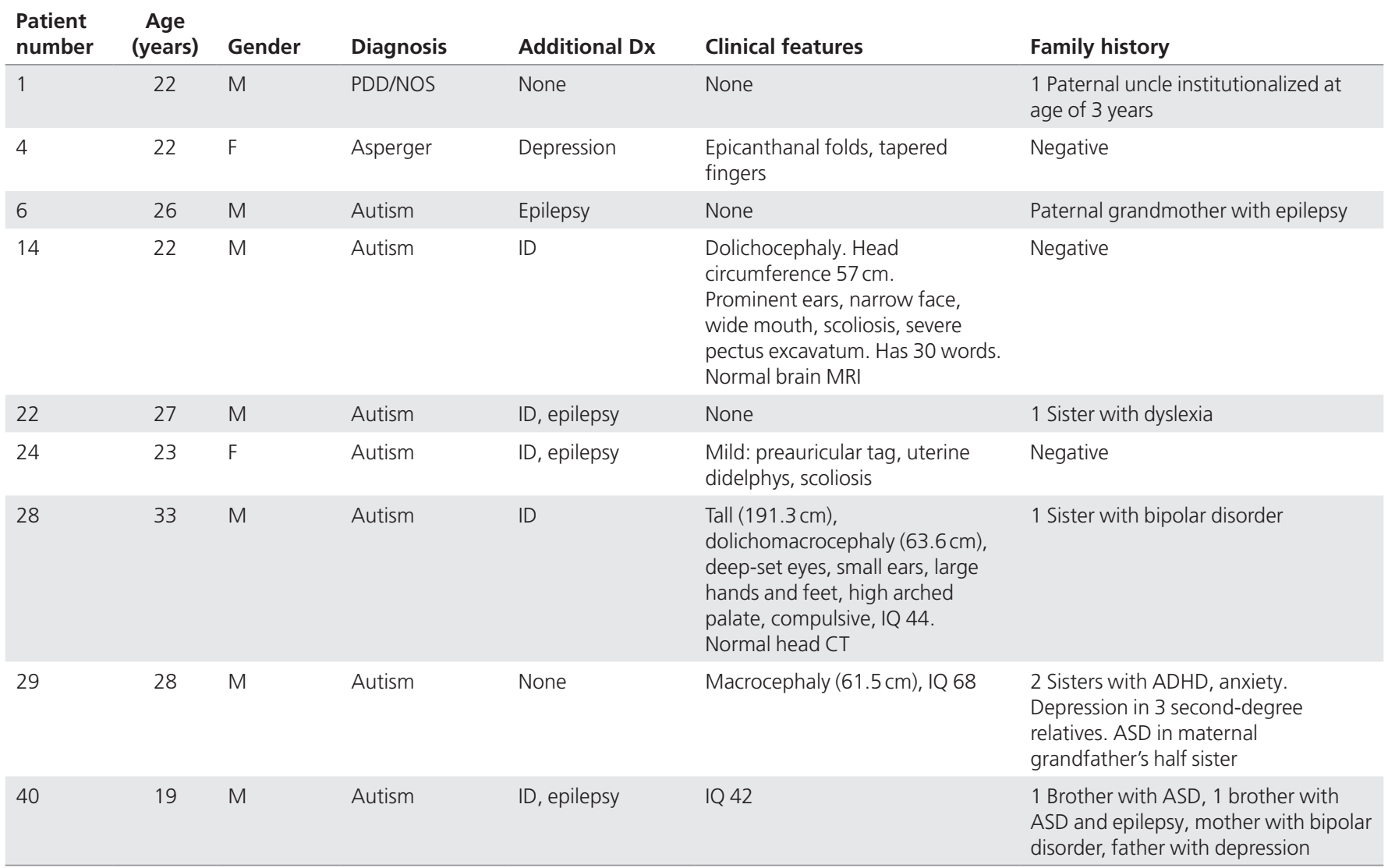

aCGH, array comparative genomic hybridization; ADHD, attention-deficit hyperactivity disorder; ASD, autism spectrum disorder; CT, computed tomography;

Dx, diagnosis; F, female; ID, intellectual disability; IQ, intelligence quotient; M, male; MRI, magnetic resonance imaging; PDD/NOS, pervasive developmental disorder; not otherwise specified; VUS, variant of uncertain significance.

Patient 1 had a 202-kb loss on 9q31.1 containing CYLC2, which encodes cylicin II, a protein component of the cytoskeletal calyx of mammalian sperm heads, unlikely to be associated with ASD. ${ }^{16}$ One deletion and two duplications in CYLC2 were reported in the DGV in the general population, and the frequency in the control population was $0.04 \%$ (Table 2). Although it is rare, given its gene function, this was classified as a VUS, likely benign. 
Patient 4 had a copy gain in the region of $4 \mathrm{q} 24$, with minimum size of $359.68 \mathrm{~kb}$. This interstitial duplication contains the C-terminal part of TET2, PPA2, and EEF1A1P9, and the N-terminal part of ARHGEF38. Therefore, it could potentially form a chimeric gene containing the N-terminal part of ARHGEF38 and the C-terminal part of TET2. TET2 encodes tet methylcytosine dioxygenase 2 , and defects in this gene have been associated with several myeloproliferative disorders. ${ }^{17}$ PPA2 encodes pyrophosphatase 2, a nuclear encoded mitochondrial protein. ${ }^{18}$ ARHGEF38 (Rho guanine nucleotide exchange factor 38) is uncharacterized. Similar-sized duplications have not been reported in DGV or dbVAR. The patient's mother was tested and was negative for the duplication; her father was not available. The clinical significance of this duplication is unknown because of a paucity of information; therefore, it was classified as a VUS.

In patient 6 (male), two CNVs were detected: a duplication of $288.05 \mathrm{~kb}$ on Xp22.13 containing BEND2 and a deletion of $45.53 \mathrm{~kb}$ on $10 \mathrm{q} 23.1$ within the intron 3 of NRG3. Similar CNVs have not been reported in DGV or dbVAR control cohorts. Little information is available for $B E N D 2$, so this was classified as a VUS. NRG3 encodes a member of the neuregulin family, which are neuronally expressed proteins in the developing nervous system. ${ }^{19}$ Neuregulin 3 is a ligand for the transmembrane tyrosine kinase receptor ERBB4, a member of the epidermal growth factor receptor family. NRG3-ERBB4 binding activates intracellular signaling cascades and the induction of cellular responses, including neuroblast proliferation, migration and differentiation. ${ }^{20,21}$ Recent studies have implicated this gene as a susceptibility locus for neuropsychiatric disorders, including schizophrenia and autism..$^{22,23}$ Although NRG3 is a compelling candidate gene for autism in the literature, because whether this intronic deletion would affect splicing or expression of NRG3 is unknown, this deletion was considered a VUS.

Patient 14 also had two CNVs. One CNV overlapped with the gene REEP5 and resulted in the deletion of its promoter region and its first two to four exons. REEP5 (also known as $D P 1)$ encodes receptor expression-enhancing protein 5 and is involved directly in shaping the tubular endoplasmic reticulum. ${ }^{24}$ Despite its important function, the dosage effect of REEP5 in humans has not been established. No similar-sized deletions were reported in DGV or dbVAR. Therefore, it was classified as a VUS. The other CNV is a duplication that contains KIAA0415, RADIL, PAPOLB, and exons 2 to 8 of FOXK1. FOXK1 encodes forkhead box K1, which regulates myogenic progenitor cell activation and muscle regeneration. ${ }^{25}$ However, it is not known if this duplication would alter FOXK1 expression or function. There is no information on KIAA0415. $R A D I L$, a ras association and dilute domains protein, has its highest expression in the adult brain, spinal cord, testis, and pancreas. RADIL is a RAS-related protein GTPase interactor and is required for cell adhesion and migration. RADIL was found to mediate migration of neural crest precursors in the zebrafish model. ${ }^{26}$ PAPOLB encodes a testis-specific poly(A) polymerase beta and regulates spermatogenesis. ${ }^{27}$ Although this duplication overlaps the much larger duplication in patient 28, and both include FOXK1, the clinical significance of this duplication is unknown; there is a similar duplication in the DGV, and its frequency in the control population was $0.01 \%$. Therefore, this duplication was classified as a VUS.

Patient 22 (male) had a duplication in the region of Xp22.33 or Yp11.32p11.31 (pseudoautosomal region 1), containing ASMT except for its first exon. ASMT encodes acetylseratonin $O$-methyltransferase, which catalyzes the last step of melatonin synthesis. Genetic variants of ASMT appear to be associated with attention-deficit and hyperactivity disorder (ADHD) and intellectual disability. ${ }^{28,29} \mathrm{~A}$ partial duplication in the ASMT gene was observed in $6-7 \%$ of the cases of ASD but in only $2 \%$ of controls $(P=0.003) .^{30}$ Smaller duplications involving exons of ASMT have been reported in 11 individuals in the DGV. Therefore, although ASMT is a compelling gene for autism, and CNVs have been reported to be enriched in autism patients, it is unknown if this duplication in the proband would disrupt ASMT function, so it was classified as a VUS.

Patient 24 (female) had a duplication on the chromosome $\mathrm{X}$ pseudoautosomal region 1 band Xp22.33/Yp11.32, containing CRLF2 and CSF2RA. Fluorescence in situ hybridization using BAC probe RP11-309M23 ruled out an insertion of this duplicated region elsewhere in the genome. A recent fine-scale survey of $\mathrm{X}$-chromosome $\mathrm{CNVs}$ verified that the duplication including CRLF2 and CSF2RA has been excluded from disease causation by segregation analysis. ${ }^{31}$ There are a few similar duplications in DGV in the general population. Therefore, this $\mathrm{CNV}$ probably represents a rare polymorphism and was classified as a VUS, likely benign.

In patient 28 , with moderate to severe intellectual disability and autism, a $4.67 \mathrm{Mb}$ duplication containing 49 genes was detected, and a similar duplication was reported in one individual in the DECIPHER database in a patient with autistic behavior, seizures, brachycephaly, high palate, short palpebral fissures, short metacarpals, and renal agenesis. Because of the size of the duplication, the number of genes in the region, and concordance with a previously reported patient, this CNV was considered a VUS, likely pathogenic.

Patient 29 has a $122-\mathrm{kb}$ loss on $7 \mathrm{q} 31.1$ containing at least the second and third exons of the IMMP2L gene. IMMP2L encodes mitochondrial inner membrane peptidase subunit 2; this complex processes the signal peptide sequences of proteins targeted to mitochondria. IMMP2L was reported previously as one of multiple candidate genes in ASD by homozygous haplotype sharing. ${ }^{32}$ Recent studies show that the inherited rare CNV-associated gene set is significantly enriched for genes reported as candidates in studies of autism, schizophrenia, Tourette syndrome, and ADHD, including A2BP1, AUTS2, CNTNAP2, and IMMP2L. ${ }^{33,34}$ Similar deletions including IMMP2L are also reported in DGV for seven individuals, and their frequency in the control population was $0.56 \%$. Deletions in IMMP2L could be associated with increased risk for autism but demonstrate reduced penetrance, similar to the chromosome 16p11.2 deletion. ${ }^{11}$ Because multiple publications have implicated IMMP2L as a candidate autism 
gene, including gene deletions, and it is rare in the general population, this CNV was classified as likely pathogenic.

In patient 40 (male), with both autism and epilepsy, a duplication of $149.27 \mathrm{~kb}$ on Xp11.21 was detected. This interstitial duplication overlaps with the C-terminal domain of the KLF8 (ZNF741) gene. Fluorescence in situ hybridization using BAC probe RP13-1021K9 showed that it was an interstitial duplication. Depending on the orientation and site of the insertion, it could potentially interrupt the KLF8 gene or change its expression. Follow-up studies showed that this CNV was inherited from the patient's mother, who has bipolar disease. The proband has two brothers with autism but not epilepsy; the copy gain was present in one and absent in the other. The autistic brother without the duplication could be a phenocopy. Their father has depression and had a normal array result. The KLF8 gene encodes a member of the Sp/KLF family of transcription factors, which contain a C-terminal DNA-binding domain with three Kruppel-like zinc fingers. The encoded protein regulates the epithelial to mesenchymal transition during development and plays a role in tumor invasion and metastasis. ${ }^{35-38}$ Similar duplications have not been reported in DGV. Moreover, a similar duplication has been reported in the DECIPHER database in a man with motor nerve abnormalities and retinal/choroid coloboma (https://decipher.sanger.ac.uk/patient/253748). A de novo balanced translocation $\mathrm{t}(\mathrm{X} ; 21)(\mathrm{p} 11.2 ; \mathrm{q} 22.3)$ that interrupted the KLF8 gene was reported in a girl with nonsyndromic mental retardation and childhood seizures. ${ }^{39}$ Analysis of her lymphoblasts showed absence of the KLF8 transcript. All of her normal X chromosomes were inactivated. ${ }^{39}$ Because of conflicting reports, our patient's duplication was considered a VUS.

\section{Statistical tests}

Among 23 adult patients with confirmed autism who underwent aCGH, CNVs were demonstrated in 9 of 23 (39.1\%) of patients, compared with $18.2 \%$ in published pediatric cases of ASDs. ${ }^{7}$ The two-tailed $P$ value calculated using Fisher's exact test was statistically significant at 0.025 , suggesting that the adult ASD population is enriched for aCGH abnormalities and rare CNVs. Among these 9 patients, pathogenic and likely pathogenic results were found in 4 of 23 (17.4\%) of patients and VUS results in 5 of 23 (21.7\%) of patients, as compared with 7 and $11.2 \%$, respectively, in a pediatric ASD cohort. ${ }^{7}$ The differences in both categories were not statistically significant due to small sample size ( $P$ values of 0.078 and 0.171 , respectively).

\section{DISCUSSION}

The use of aCGH as a first-line diagnostic test in the evaluation of patients with autism has been supported by the American College of Medical Genetics and Genomics, and the International Standard Cytogenomic Array Consortium. ${ }^{6,9}$ Published studies were performed using entirely or largely pediatric populations, and the yield and types of abnormalities in adult populations have not been studied. We report here on an adult population of consecutively evaluated clinic patients referred for genetic evaluation of ASDs. We found that the yield of genetic abnormalities in an adult population was $39.1 \%$, significantly higher than previously described in the pediatric ASD population (18.2\%), $P$ value 0.025 . Our study found that genomic losses and gains are compatible with survival into adulthood.

The identification of a specific diagnosis was welcome information for families, and some families expressed gratitude for the opportunity for updated genetic evaluation even in the absence of a diagnostic finding. Although one reason for attempting to identify the genetic cause of an ASD in childhood is to offer accurate reproductive recurrence risk counseling to parents, there is an equal and perhaps greater reason to consider identification of the cause of ASD in young adults in order to provide risk counseling to their siblings of reproductive age, because any individual has only two parents but can have more than two siblings. Indeed, diagnosis for reproductive risk to sibs was a commonly stated goal of genetic counseling for families of this young adult population. Our patients were relatively disabled according to their living situation and employment history, but some patients were living independently or semi-independently, and may wish to know their own reproductive risk.

Our study was limited by the size of the study population and potential referral bias as to which patients were referred for genetic evaluation. However, the demographics of the study cohort reflect the national autism spectrum population in some aspects. The sex ratio was 3.5 men to 1 woman, and $83 \%$ had completed high school. Of 16 patients who had previously undergone metabolic or cytogenetic testing, testing for single gene disorders, neuroimaging, and muscle or skin biopsy, only one had had a diagnostic result. Of the 24 autism patients who underwent updated genetic testing, 1 of 24 (4\%) had a new diagnosis of fragile $\mathrm{X}$ syndrome, and of patients who underwent aCGH testing, 2 of $23(8.7 \%)$ received a new diagnosis of either Smith-Magenis syndrome or presumed pathogenic15q11.2-q13 duplication syndrome, and 2 patients had VUSs considered likely pathogenic (see Tables 1 and 2). The overall yield of diagnostic abnormalities or CNVs likely pathogenic was 5 of 24 or $21 \%$.

Given our results, we recommend repeat genetic evaluation for adults with ASDs. Nationwide, there are fewer genetics clinics and services for adult patients (other than cancer genetics services) as compared to pediatric genetic clinics, and less awareness among health-care providers of the benefits of genetic evaluation for adults.

Our study demonstrates the value of clinical genetic evaluation in an adult population with known or suspected ASDs. The presence of three or more minor anomalies has been considered significant because of the increased risk of major malformations, and this was used to classify patients as dysmorphic. ${ }^{40}$ This study found a significant likelihood of detecting a diagnostic abnormality in an adult patient when a potential syndrome was raised by a clinical geneticist, because fragile $\mathrm{X}$ syndrome, Smith-Magenis syndrome, and Velo-cardio-facial syndrome were all recognized clinically prior to genetic test results.

This study provides information for clinicians regarding genetic testing for adults with ASDs. Although VUSs 
outnumbered diagnostic findings, this result is similar to those of other published studies and reflects current technology and available information in databases. ${ }^{7}$ It also reinforces the need for careful genetic counseling in interpretation. Identification of genetic abnormalities can inform medical treatment and health screening for individuals with ASDs and provide information for family planning for their relatives.

\section{ACKNOWLEDGMENTS}

We thank Michael J. Guralnick, PhD, for support of the Center on Human Development and Disabilities Autism Genetics Clinic and Rosalind Oti, PhD, for reviewing neuropsychological testing data.

\section{DISCLOSURE}

The authors declare no conflict of interest.

\section{REFERENCES}

1. Kogan MD, Blumberg SJ, Schieve LA, et al. Prevalence of parent-reported diagnosis of autism spectrum disorder among children in the US, 2007. Pediatrics 2009;124:1395-1403.

2. Autism and Developmental Disabilities Monitoring Network Year 2008 Principal Investigators. Prevalence of autism spectrum disorders-Autism and Developmental Disabilities 14 Sites, United States. MMWR Surveill Summ 2012;61;1-19.

3. Shaffer LG, Kashork CD, Saleki R, et al. Targeted genomic microarray analysis for identification of chromosome abnormalities in 1500 consecutive clinical cases. J Pediatr 2006;149:98-102.

4. de Vries BB, Pfundt R, Leisink $M$, et al. Diagnostic genome profiling in mental retardation. Am J Hum Genet 2005;77:606-616.

5. Rauch A, Rüschendorf F, Huang J, et al. Molecular karyotyping using an SNP array for genomewide genotyping. J Med Genet 2004;41:916-922.

6. Miller DT, Adam MP, Aradhya S, et al. Consensus statement: chromosomal microarray is a first-tier clinical diagnostic test for individuals with developmental disabilities or congenital anomalies. Am J Hum Genet 2010;86:749-764.

7. Shen Y, Dies KA, Holm IA, et al.; Autism Consortium Clinical Genetics/DNA Diagnostics Collaboration. Clinical genetic testing for patients with autism spectrum disorders. Pediatrics 2010;125:e727-e735.

8. Taylor MR, Jirikowic J, Wells C, et al. High prevalence of array comparative genomic hybridization abnormalities in adults with unexplained intellectual disability. Genet Med 2010;12:32-38.

9. Manning M, Hudgins L; Professional Practice and Guidelines Committee. Array-based technology and recommendations for utilization in medical genetics practice for detection of chromosomal abnormalities. Genet Med 2010;12:742-745.

10. Kearney HM, Thorland EC, Brown KK, Quintero-Rivera F, South ST; Working Group of the American College of Medical Genetics Laboratory Quality Assurance Committee. American College of Medical Genetics standards and guidelines for interpretation and reporting of postnatal constitutional copy number variants. Genet Med 2011;13:680-685.

11. Weiss LA, Shen Y, Korn JM, et al.; Autism Consortium. Association between microdeletion and microduplication at 16p11.2 and autism. N Engl J Med 2008;358:667-675.

12. Miller DT, Nasir R, Sobeih MM, Shen Y, Wu B-L, Hanson E. 16p11.2 Microdeletion. 2009 Sep 22 [Updated 27 October 2011]. In: Pagon RA, Bird TD, Dolan CR, et al. (eds). GeneReviews ${ }^{\mathrm{TM}}$ [Internet]. University of Washington, Seattle: Seattle, WA, 1993. http://www.ncbi.nlm.nih.gov/books/NBK11167/. Accessed 23 September 2012.

13. Cook EH Jr, Lindgren V, Leventhal BL, et al. Autism or atypical autism in maternally but not paternally derived proximal $15 q$ duplication. Am J Hum Genet 1997;60:928-934.

14. Wu DJ, Wang NJ, Driscoll J, et al. Autistic disorder associated with a paternally derived unbalanced translocation leading to duplication of chromosome 15pter-q13.2: a case report. Mol Cytogenet 2009; 2:27.

15. Cooper GM, Coe BP, Girirajan S, et al. A copy number variation morbidity map of developmental delay. Nat Genet 2011;43:838-846.
16. Hess $\mathrm{H}$, Heid H, Zimbelmann R, Franke WW. The protein complexity of the cytoskeleton of bovine and human sperm heads: the identification and characterization of cylicin II. Exp Cell Res 1995;218:174-182.

17. Shide K, Kameda T, Shimoda H, et al. TET2 is essential for survival and hematopoietic stem cell homeostasis. Leukemia 2012;26:2216-2223.

18. Curbo S, Lagier-Tourenne C, Carrozzo R, et al. Human mitochondrial pyrophosphatase: CDNA cloning and analysis of the gene in patients with mtDNA depletion syndromes. Genomics 2006;87:410-416.

19. Carteron C, Ferrer-Montiel A, Cabedo H. Characterization of a neural-specific splicing form of the human neuregulin 3 gene involved in oligodendrocyte survival. J Cell Sci 2006;119(pt 5):898-909.

20. Zhang D, Sliwkowski MX, Mark M, et al. Neuregulin-3 (NRG3): a novel neural tissue-enriched protein that binds and activates ErbB4. Proc Natl Acad Sci USA 1997;94:9562-9567.

21. Kao WT, Wang Y, Kleinman JE, et al. Common genetic variation in Neuregulin 3 (NRG3) influences risk for schizophrenia and impacts NRG3 expression in human brain. Proc Natl Acad Sci USA 2010;107:15619-15624.

22. Britsch $\mathrm{S}$. The neuregulin-//ErbB signaling system in development and disease. Adv Anat Embryol Cell Biol 2007;190:1-65.

23. Lin M, Hrabovsky A, Pedrosa E, Wang T, Zheng D, Lachman HM. Allele-biased expression in differentiating human neurons: implications for neuropsychiatric disorders. PLOS ONE 2012;7:e44017

24. Voeltz GK, Prinz WA, Shibata Y, Rist JM, Rapoport TA. A class of membrane proteins shaping the tubular endoplasmic reticulum. Cell 2006;124:573-586. Erratum in: Cell 2007;130:754.

25. Shi X, Bowlin KM, Garry DJ. Fhl2 interacts with Foxk1 and corepresses Foxo4 activity in myogenic progenitors. Stem Cells 2010;28:462-469.

26. Smolen GA, Schott BJ, Stewart RA, et al. A Rap GTPase interactor, RADIL, mediates migration of neural crest precursors. Genes Dev 2007;21:2131-2136.

27. Kashiwabara S, Noguchi J, Zhuang T, et al. Regulation of spermatogenesis by testis-specific, cytoplasmic poly(A) polymerase TPAP. Science 2002;298: 1999-2002.

28. Chaste P, Clement N, Botros HG, et al. Genetic variations of the melatonin pathway in patients with attention-deficit and hyperactivity disorders. J Pineal Res 2011;51:394-399.

29. Pagan C, Botros HG, Poirier K, et al. Mutation screening of ASMT, the last enzyme of the melatonin pathway, in a large sample of patients with intellectual disability. BMC Med Genet 2011;12:17.

30. Cai G, Edelmann L, Goldsmith JE, et al. Multiplex ligation-dependent probe amplification for genetic screening in autism spectrum disorders: efficient identification of known microduplications and identification of a novel microduplication in ASMT. BMC Med Genomics 2008;1:50.

31. Whibley AC, Plagnol V, Tarpey PS, et al. Fine-scale survey of $X$ chromosome copy number variants and indels underlying intellectual disability. Am J Hum Genet 2010;87:173-188.

32. Casey JP, Magalhaes T, Conroy JM, et al. A novel approach of homozygous haplotype sharing identifies candidate genes in autism spectrum disorder. Hum Genet 2012;131:565-579.

33. Elia J, Gai X, Xie HM, et al. Rare structural variants found in attention-deficit hyperactivity disorder are preferentially associated with neurodevelopmental genes. Mol Psychiatry 2010;15:637-646.

34. Maestrini E, Pagnamenta AT, Lamb JA, et al.; IMGSAC. High-density SNP association study and copy number variation analysis of the AUTS1 and AUTS5 loci implicate the IMMP2L-DOCK4 gene region in autism susceptibility. Mol Psychiatry 2010;15:954-968.

35. van Vliet J, Turner J, Crossley M. Human Krüppel-like factor 8: a CACCC-box binding protein that associates with CtBP and represses transcription. Nucleic Acids Res 2000;28:1955-1962.

36. Eaton SA, Funnell AP, Sue N, Nicholas H, Pearson RC, Crossley M. A network of Krüppel-like Factors (Klfs). Klf8 is repressed by Klf3 and activated by Klf1 in vivo. J Biol Chem 2008;283:26937-26947.

37. Suske G, Bruford E, Philipsen S. Mammalian SP/KLF transcription factors: bring in the family. Genomics 2005:85:551-556.

38. Wang $X$, Zheng $M$, Liu G, et al. Krüppel-like factor 8 induces epithelial to mesenchymal transition and epithelial cell invasion. Cancer Res 2007;67:71847193

39. Lossi AM, Laugier-Anfossi F, Depetris $D$, et al. Abnormal expression of the KLF8 (ZNF741) gene in a female patient with an $X$; autosome translocation $\mathrm{t}(\mathrm{X} ; 21)(\mathrm{p} 11.2 ; \mathrm{q} 22.3)$ and non-syndromic mental retardation. J Med Genet 2002:39:113-117.

40. Leppig KA, Werler MM, Cann Cl, Cook CA, Holmes LB. Predictive value of minor anomalies. I. Association with major malformations. J Pediatr 1987;110: 531-537. 